\title{
Efficient creation of maximally entangled states by modulation of tunneling rates
}

\author{
Gentaro Watanabe ${ }^{1,2}$ \\ ${ }^{1}$ RIKEN, 2-1 Hirosawa, Wako, Saitama 351-0198, Japan \\ ${ }^{2}$ CNR INFM-BEC and Department of Physics, University of Trento, I-38050 Povo, Italy
}

(Dated: November 10, 2018)

\begin{abstract}
For systems described by the two-site Bose-Hubbard Hamiltonian, we show that a sinusoidal modulation of the tunneling matrix element assists higher-order co-tunneling processes. Using this mechanism, we propose an efficient new scheme for creating a coherent superposition of states in which all particles are either on one site or all on the other site, the so-called NOON state. This scheme yields an almost perfect NOON state periodically. For larger numbers of particles, further reduction of the time to create the state is possible if more than one modulation frequency is employed. With this scheme, NOON states with a larger number of particles could be realized with state-of-the-art techniques for cold Bose gases in a double-well potential.
\end{abstract}

PACS numbers: 03.75.Lm, 03.75.Gg, 42.50.Dv, 03.65.Xp

One of the current challenges in quantum control is to create particular sorts of entangled states that are a superposition of states that are maximally different. The largest number of particles for which such states can be engineered is six for ions in traps [1] and $\approx 10$ photons [2] for photons. Atoms in a double-well potential are a fundamental system for quantum-state engineering and it is a promising candidate for creating such superposition states. Especially the coherent superposition of a state in which all $N$ particles are in the right well and that in which all particles are in the left well (a so-called NOON state [3]) is a maximally entangled state [4, 5], and it is an important resource for matter-wave interferometry: it provides the ultimate quantum limit of the phase resolution $\sim 1 / N$, i.e., the Heisenberg limit, for any $N[6]$ (see also [7]). However, NOON states with larger $N$ are fragile with respect to decoherence and thus it is crucial to create them in a short time.

In Ref. [8], we studied the tunneling of bosons in a double-well potential [9] described by the two-site BoseHubbard Hamiltonian [10]:

$$
\hat{H}=-J\left(\hat{c}_{R}^{\dagger} \hat{c}_{L}+\hat{c}_{L}^{\dagger} \hat{c}_{R}\right)+\frac{U}{2}\left(\hat{c}_{R}^{\dagger} \hat{c}_{R}^{\dagger} \hat{c}_{R} \hat{c}_{R}+\hat{c}_{L}^{\dagger} \hat{c}_{L}^{\dagger} \hat{c}_{L} \hat{c}_{L}\right)
$$

where $\hat{c}_{R}^{\dagger}$ and $\hat{c}_{L}^{\dagger}$ create bosons in the right and left well, respectively, $J$ is the tunneling matrix element, and $U$ is the on-site interaction. There, we found that, starting from a situation in which all particles are in the right or the left well, a NOON state is formed by co-tunneling provided the interaction is strong enough: $\kappa / 2 \equiv U N / 2 J \gg 1$. In this situation, single-particle tunneling is suppressed by the energy conservation and all $N$ particles are forced to be in the same well. While the classical theory predicts self-trapping [11, 12], a superposition of all particles in the right and left wells is allowed in quantum mechanics and an oscillation between these two states occurs due to higher-order co-tunneling, which yields a NOON state after a quarter of the oscillation period [8]. Indeed, a NOON state for $N=2$ would have been realized by this mechanism in experiments of Ref. [13]. A great advantage of this mechanism is that almost perfect NOON states are obtained at periodic intervals in double-well potentials unlike, e.g., the well-known protocol of Ref. [14] (see also [15, 16] and references therein; protocols for different setups are described in, e.g., Refs. [17, 18]). On the other hand, a serious difficulty with the mechanism of Ref. [8] is that the time to form NOON states is much larger than for other protocols and increases exponentially with $N$. In the present work, we shall show how this drawback may be overcome. We also show that, using our present scheme, NOON states with $N$ comparable to the largest value attained for trapped ions [1] can be realized with cold atoms under state-ofthe-art experimental conditions [13].

Let us first evaluate the energy splitting $\Delta E_{\Delta N}$ of the two degenerate states with the same value of $|\Delta N| \equiv$ $\left|N_{R}-N_{L}\right|$, where $N_{R}$ and $N_{L}$ are the numbers of particles in the right and left wells, respectively. The onsite interaction term, which reads $\hat{H}_{0} \equiv U\left(\hat{c}_{R}^{\dagger} \hat{c}_{R}^{\dagger} \hat{c}_{R} \hat{c}_{R}+\right.$ $\left.\hat{c}_{L}^{\dagger} \hat{c}_{L}^{\dagger} \hat{c}_{L} \hat{c}_{L}\right) / 2=U \Delta \hat{N}^{2} / 4+U\left(\hat{N}^{2}-2 \hat{N}\right) / 4$, gives the zeroth-order result for the energy as $E_{\Delta N}^{(0)} \equiv U \Delta N^{2} / 4$ (see Fig. 1). Here $\hat{N} \equiv \hat{c}_{R}^{\dagger} \hat{c}_{R}+\hat{c}_{L}^{\dagger} \hat{c}_{L}$ and $\Delta \hat{N} \equiv$ $\hat{c}_{R}^{\dagger} \hat{c}_{R}-\hat{c}_{L}^{\dagger} \hat{c}_{L}$. The splitting $\Delta E_{\Delta N}$ can be calculated by $|\Delta N|$ th-order perturbation theory treating the hopping term as a perturbation and we obtain

$$
\Delta E_{\Delta N}=2 J N \kappa^{-|\Delta N|+1} \beta(N, \Delta N),
$$

with

$$
\beta(N, \Delta N) \equiv \frac{[(N+|\Delta N|) / 2] ! N^{|\Delta N|-2}}{[(N-|\Delta N|) / 2] ![(|\Delta N|-1) !]^{2}} .
$$

The tunneling period $T_{\Delta N}$ between these states is given by $T_{\Delta N}=2 \pi \hbar / \Delta E_{\Delta N}$. Observe that, for $\kappa \gg 1, T_{\Delta N}$ grows exponentially with $|\Delta N|$.

Our basic idea for reducing the formation time of the NOON state is to modulate the tunneling matrix element we make a resonance between the states of $|\Delta N|=N$ and $|\Delta N|=N-2$ (see Fig. 11), and take advantage of the tunneling between the two states of $|\Delta N|=N-2$ whose period $T_{N-2}$ is much shorter than $T_{N}$. Using 


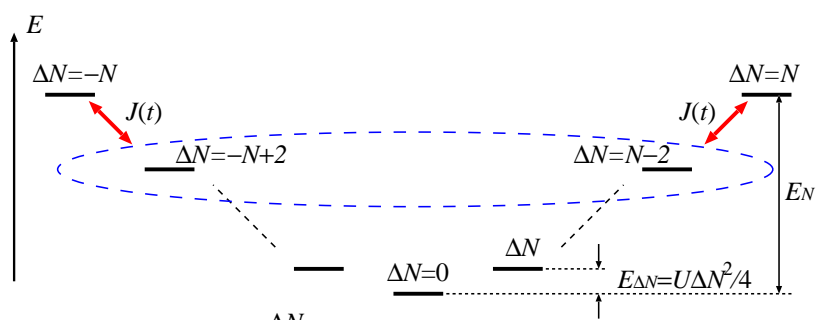

$\Delta N$

FIG. 1: (Color online) Schematic diagram of the present protocol for reducing the formation time of the NOON state.

Eqs. (2) and (3), we estimate the reduction ratio as $T_{N} / T_{N-2}=\kappa^{2}[(N-1)(N-2)]^{2} / N^{3} \sim \kappa^{2} N$. For, e.g., $N=5$ and $U / J=4$, the reduction ratio is $T_{N} / T_{N-2}=$ 460.8. From the general point of view, this mechanism in which the higher-order co-tunneling is assisted by the time-dependent tunneling matrix element would also be relevant to other contexts such as in Josephson qubits (e.g., [19] for review). Moreover, our theory directly applies also to another situation: cold Bose gases in two hyperfine states coupled by an external pumping field (see, e.g., [15]). In this case, $J$ corresponds to the Rabi frequency of the external field and by controlling its intensity one can change $J$.

We consider sinusoidal modulation of the tunneling matrix element and employ the two-site Bose-Hubbard Hamiltonian (1) with $J$ replaced by $J(t)$ where

$$
J(t) \equiv J(1+A \sin \omega t) .
$$

Here $A$ and $\omega$ are the amplitude and the frequency of the modulation, respectively. This modulation can be realized by controlling the height of the barrier in the case of the double-well potential (see, e.g., Ref. 20] for reproducing $J(t)$ of Eq. (4) in three-dimensional doublewell potentials). The condition for a resonance between the states with $|\Delta N|=N$ and $|\Delta N|=N-2$ is

$$
\hbar \omega \simeq E_{N}^{(0)}-E_{N-2}^{(0)}=U(N-1) .
$$

As we shall see later, to obtain the periodic dynamics, the modulation frequency must be much larger than the frequency of tunneling oscillations, $\omega \gg 2 \pi / T(\omega)$.

Now we calculate the time evolution using the two-site Bose-Hubbard Hamiltonian with $J(t)$ of Eq. (4). Here we take $\left|N_{L}, N_{R}\right\rangle=|0, N\rangle$ as an initial condition. In Fig. 2, we show the time evolution of $\langle\Delta N\rangle / N$ [panel (a)] and its fluctuation $\sigma_{\Delta N} \equiv \sqrt{\left\langle\Delta N^{2}\right\rangle-\langle\Delta N\rangle^{2}}$ [panel (b)] for $N=5, U / J=4, A=0.1$, and $\hbar \omega / J=15$ 21] as an example. Resulting dynamics has a periodic nature with a period $T \simeq 88 T_{0}$, which is much shorter than that without modulation evaluated by Eqs. (2) and (3): $T=1228.8 T_{0}$. Here $T_{0} \equiv 2 \pi / \omega_{0}=\pi \hbar / J$, where $\omega_{0} \equiv 2 J / \hbar$ and $T_{0}$ are the oscillation frequency and the period of the tunneling for $U=0$. We also see that $\sigma_{\Delta N}$ almost reaches $\sigma_{\Delta N}=N=5$ when $\langle\Delta N\rangle / N=0$ corresponding to a NOON state. Figure 2(c) shows the
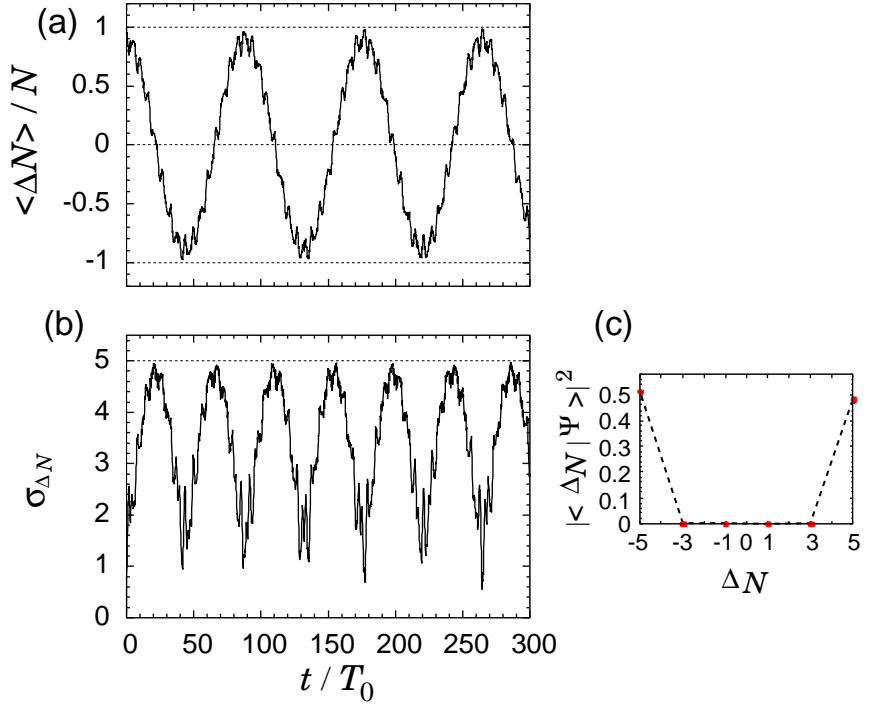

FIG. 2: (Color online) Time evolution of $\langle\Delta N\rangle / N$ (a) and its fluctuation $\sigma_{\Delta N}$ (b) for $N=5, U / J=4, A=0.1$, and $\hbar \omega / J=15$. The tunneling period is $T \simeq 88 T_{0}$. Panel (c) shows the population of each component of $|\Delta N\rangle$ at $t=285.27 T_{0}$.

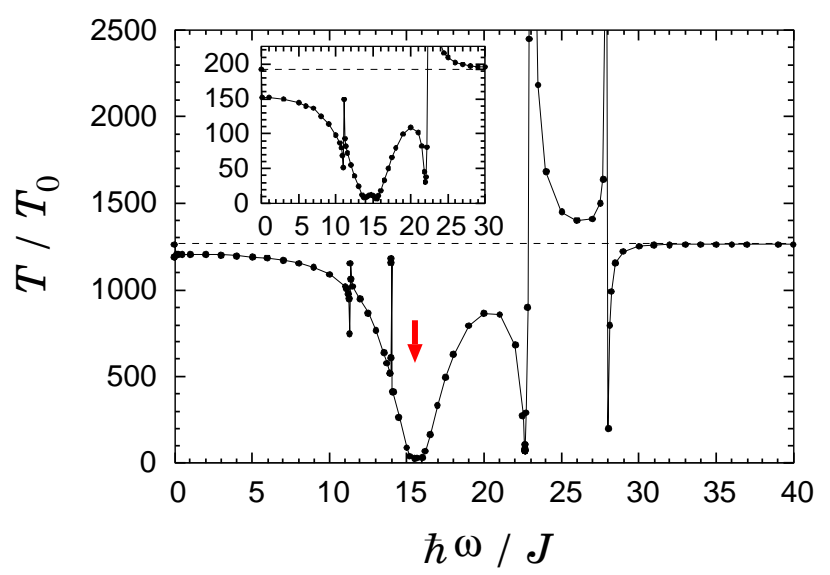

FIG. 3: (Color online) The tunneling period $T$ as a function of $\omega$ for $N=5, U / J=4$, and $A=0.1$. There is a drastic reduction of $T$ in a wide range around $\hbar \omega / J \simeq 16$ (red arrow). The dashed lines show $T$ without modulation. Very narrow resonances in the low- $\omega$ region of $\hbar \omega / J \lesssim 10$ are not shown. The inset shows for $N=4, U / J=5$, and $A=0.3$.

snapshot of the population of each component of $|\Delta N\rangle$ at $t=285.27 T_{0}$; here, we have an almost perfect NOON state of $\sigma_{\Delta N}=4.994$.

In Fig. 3. we show the resulting tunneling period $T$ as a function of $\omega$ for the same parameters as in Fig. 2. The resonance condition (5) gives $\hbar \omega / J=16$. We note that there is a dramatic reduction of $T$ around $\hbar \omega / J=16$ with a wide width in $\omega$ (red arrow) 22]. Let us first discuss the high- and low- $\omega$ regimes. In the high- $\omega$ region of $\hbar \omega / J \gtrsim 30$, tunneling period does not depend on $\omega$ any more and is almost the same as in the case without 


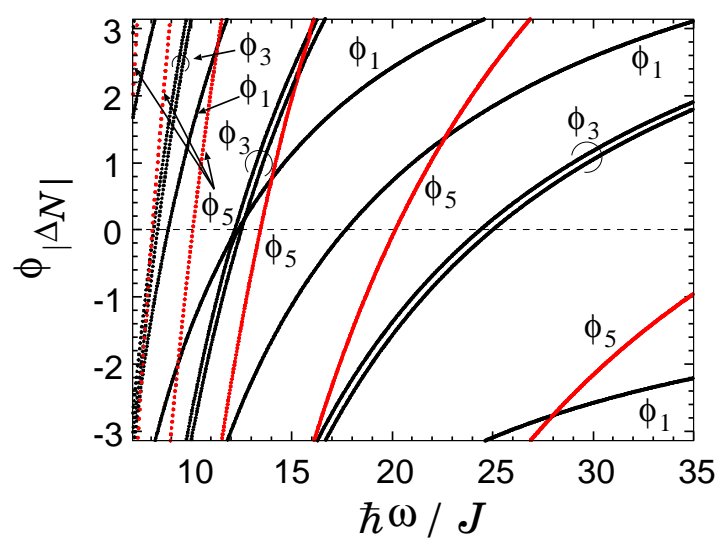

FIG. 4: (Color online) Eigenvalues $\phi_{|\Delta N|}$ of the Floquet operator as functions of $\hbar \omega / J$ for the same parameters as in Fig. 3 . $N=5, U / J=4$, and $A=0.1$. The red points (they look like lines except for the low- $\omega$ region) show $\phi_{5}$ and two sets of the red lines almost overlap. Resonance occurs at $\omega$ where $\phi_{5}$ (red lines) crosses the other $\phi_{|\Delta N|}$; i.e., at $\hbar \omega / J \simeq 11.3,14.0,15.5$ $16,22.6$, and 28.0.

modulation. This is simply because $\hbar \omega / J \gtrsim 30$ is bigger than any other energy scales in this problem and thus the system is insensible to the modulation of $\hbar \omega / J \gtrsim 30$. In the low $\omega$ region of $\hbar \omega / J \lesssim 10$ (but $\omega$ being much larger than the frequency of the tunneling oscillation), $\omega$ dependence of $T$ is weak (except for very narrow resonances, which are not shown in this figure). Since $2 \pi / \omega \ll T$ even though $\omega$ is small, the tunneling period can be evaluated by the time average of Eq. (2) with replacing $J$ by $J(t)$ of Eq. (4): $T_{\text {low } \omega}=2 \pi /\left\langle\Delta E_{N, J(t)}\right\rangle_{t}$ with $\left\langle\Delta E_{N, J(t)}\right\rangle_{t} \equiv\left\langle 2 J(t) N(J(t) / U N)^{N-1}\right\rangle_{t} \beta(N, N)=$ $2 J N \kappa^{-N+1} \beta(N, N)\left\langle(1+A \sin \omega t)^{N}\right\rangle_{t}$. This replacement of $J^{N}$ by $\left\langle J^{N}(t)\right\rangle_{t}$ yields an effective increase of the energy splitting and thus a reduction of $T$.

Next, we discuss the narrow resonances observed in Fig. 3. For this purpose, Floquet theory analysis is useful (e.g., Refs. [20, 23]). The Floquet operator $\hat{F}$ is a mapping between the state at $t_{0}$ and the state after one modulation period at $t_{0}+2 \pi / \omega:\left|\Psi\left(t_{0}+2 \pi / \omega\right)\right\rangle=\hat{F}\left|\Psi\left(t_{0}\right)\right\rangle$. Here we get $\hat{F}$ as follows. Starting from each state of the basis set $\{|\Delta N\rangle ; \Delta N=-N,-N+2, \ldots, N\}$, we follow the time evolution for one modulation period, $2 \pi / \omega$. Each resulting state forms a column of the matrix of $\hat{F}$.

In Fig. 4, we show the eigenvalues of $\hat{F}$ as functions of $\hbar \omega / J$ for the same parameters as in Fig. 3. We denote the eigenvalue of $\hat{F}$ as $\phi_{|\Delta N|}$ whose eigenstate has maximum amplitude at $\pm \Delta N$ with even or odd parity in the Fock space labeled by $\Delta N$ (However, there are significant populations in the other components. They become larger when two eigenvalues have a crossing in Fig. 4.). In this figure, the red points (they look like lines except for the low- $\omega$ region) show $\phi_{5}$ and two sets of the red lines are so close that they appear as a single line. By comparing with Fig. 3, we observe that the resonances occur

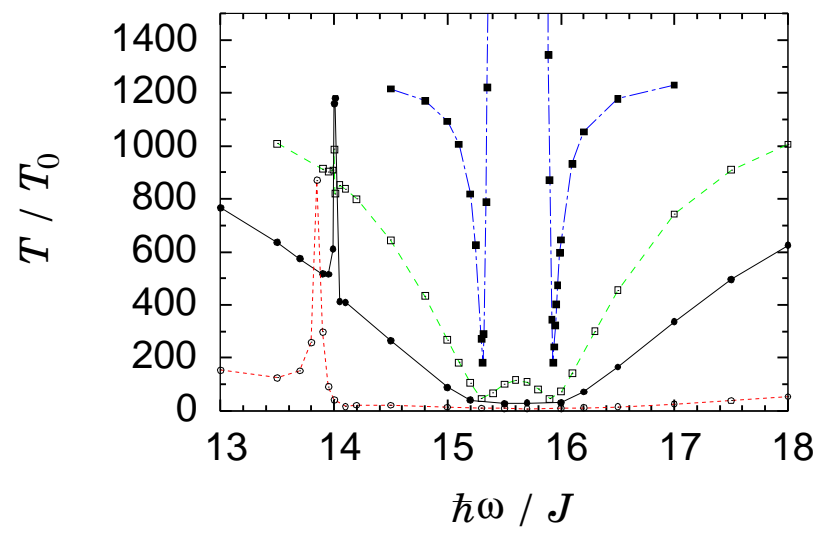

FIG. 5: (Color online) The tunneling period $T$ around $\hbar \omega / J=16$ as a function of $\hbar \omega / J$ for various values of $A$. The other parameters are the same as in Figs. 2 and 3. $N=5$ and $U / J=4$. From the lower to the higher lines, $A=0.5$ (dotted line), 0.1 (solid line), 0.05 (dashed line), and 0.01 (dashed-dotted line).

when $\phi_{5}$ (red lines) crosses the other $\phi_{|\Delta N|}$ (black lines): the narrow resonances occur at $\hbar \omega / J \simeq 11.3,14.0,22.6$, and 28.0 , where $\phi_{5}$ crosses $\phi_{1}$, and the wide one occurs at $\hbar \omega / J \simeq 15.5-16$, where $\phi_{5}$ almost simultaneously crosses two sets of $\phi_{3}$.

To understand the difference between the wide and narrow resonances, we also show the tunneling period $T$ around $\hbar \omega / J=16$ for various $A$ in Fig [5] With decreasing $A$, the resonance becomes narrower and finally it separates into two narrow resonances (see the case of $A=0.01$ ). Note that the separation of these two resonances is $\hbar \omega / J \simeq 0.75$, corresponding to the energy splitting $\Delta E_{3}$ between the two states of $\Delta N= \pm 3$. This fact shows that, to obtain the wide resonance as in the case of $A=0.5$ and 0.1 (and 0.05) in Fig. [5] it is necessary to couple the two states of $|\Delta N|=N-2$, whose energies are different by $\Delta E_{N-2}$, to the states of $|\Delta N|=N$ (whose energy splitting is negligibly small). Thus, the energy scale of the amplitude of the modulation in the hopping term of Eq. (1) should be comparable to or larger than the energy splitting of the states of $|\Delta N|=N-2: 2 J A N \gtrsim \Delta E_{N-2}$. From Eqs. (2) and (3), we then obtain $A \gtrsim \Delta E_{N-2} / 2 J N=$ $N^{-1}(J / U)^{N-3}(N-1)(N-2) /(N-3)$ !. Concerning this point, we have some remarks. For $N=3$, this condition reads $A \gtrsim 2 / 3$, which might be too large to be realized in a real double-well potential. For $N=2$, there is only one state of $\Delta N=N-2=0$, and thus we never have a wide resonance. On the other hand, for larger $N$, further reduction of $T$ is possible by employing additional modulations corresponding to wide resonances between the states of $|\Delta N|=N-2$ and $N-4, N-4$ and $N-6$, etc., whose energy splittings can be very small for larger $N$ [see Eq. (2)] 24]. This "scalability" may be useful for creating NOON states with larger $N$.

Note also that a tilt $\hat{V}_{\text {tilt }} \equiv \Delta V \Delta \hat{N} / 2$ of the double- 
well potential, which may be caused by the imperfection of the double well or by the superimposed trapping, suppresses the tunneling [8, 25, 26]. To obtain the NOON state using the present scheme, the tilt should be smaller than the energy splitting for $|\Delta N|=N-2$ : $\Delta V N \ll \Delta E_{N-2}$.

It is useful to know the width $\Gamma$ of the wide resonance, which enables us to evaluate the formation time for a given $\omega$. We numerically obtain $\Gamma$ by the Lorentzian fit of the following form: $T=\left(T_{N}-\right.$ $\left.T_{N-2}\right)\left(1-\Gamma^{2}\left[\left(\omega-\omega_{0}\right)^{2}+\Gamma^{2}\right]^{-1}\right)+T_{N-2}$. This formula is constrained to reproduce $T=T_{N-2}$ at $\omega=\omega_{0}$ [27] and $T=T_{N}$ in the limit of $\omega \rightarrow \infty$. We find that $\Gamma$ is almost proportional to $A$ and $U$, respectively. From the results of $N \lesssim 6$, we obtain the scaling relation as $\Gamma \simeq 0.49 A U(N-1)(N-2)$.

Finally, we discuss the feasibility of creating larger NOON states in current experiments [13]. In the present situation with $N>2$, most serious decoherence process is due to three-body losses. Let us estimate the decoherence time assuming the Gaussian wave function in each well, $\psi(\mathbf{r})=\left(N / \pi^{3 / 2} d_{\perp}^{2} d_{z}\right)^{1 / 2} \exp \left[-\left(r_{\perp}^{2} / 2 d_{\perp}^{2}\right)-\left(z^{2} / 2 d_{z}^{2}\right)\right]$, where we take the direction of the double well in the $z$ direction and $d_{\perp}$ and $d_{z}$ are the transverse and the longitudinal oscillator lengths, respectively. With the threebody rate constant $K_{3}$, the decoherence time $\tau_{3}$ is given by $\tau_{3}^{-1}=K_{3} \int d^{3} r|\psi|^{6}=(\sqrt{3} \pi)^{-3} K_{3} N^{3}\left(d_{\perp}^{4} d_{z}^{2}\right)^{-1} \simeq$ $(4 \pi / 3)^{3} K_{3} N^{3} s_{\perp} s_{z}^{1 / 2}\left(\lambda_{\perp}^{2} \lambda_{z}^{4}\right)^{-1}$. Here $\lambda_{z}$ and $\lambda_{\perp}$ are the wavelengths of the lasers in the longitudinal and transverse directions, respectively, and $s_{\perp}$ and $s_{z}$ are the longitudinal and transverse lattice heights, respectively, in units of the recoil energy in the longitudinal direction
[28].

In the experiment of Ref. [13], $\lambda_{z}=765 \mathrm{~nm}, \lambda_{\perp}=843$ $\mathrm{nm}, s_{z} \simeq 10($ for $U / J=5), s_{\perp}=33$, and $K_{3}=$ $5.8 \times 10^{-42} \mathrm{~Hz} \mathrm{~m}^{6}$ for ${ }^{87} \mathrm{Rb}$ [29], and we obtain $\tau_{3} \simeq 16$ $\mathrm{ms}$ for $N=4$. Using the present scheme, the tunneling period for $N=4$ and $U / J=5$ 30] can be reduced to $\lesssim 20 T_{0}$ (see inset of Fig. 3), corresponding to $\lesssim 14.4 \mathrm{~ms}$ in this experiment. Thus, the system safely undergoes one period of the oscillation in the decoherence time. By the same technique used in Ref. [13], one can measure $\langle\Delta N\rangle$. Its oscillatory behavior is a firm evidence for the coherency of the system and rules out the possibility of the 50:50 mixture of $|N, 0\rangle$ and $|0, N\rangle$. From the oscillation period of $\langle\Delta N\rangle$, one can also reject the possibility of $|N / 2, N / 2\rangle$ at $\langle\Delta N\rangle=0$. The combination of the above two evidences establishes the realization of a NOON state.

In conclusion, we have found the higher-order cotunneling can be assisted by sinusoidally modulating the tunneling matrix element. Using this mechanism, we have proposed an efficient scheme for periodically creating almost perfect NOON states in two-state Bose systems. This scheme is scalable in a sense that, for larger number of particles, further reduction is possible using more than one frequency. With this scheme, a NOON state of four particles can be realized in current experiments of cold Bose gases in a double-well potential.

The author is grateful to Augusto Smerzi and Chris Pethick. He also thanks L. D. Carr, P. Hyllus, F. Piazza, W. P. Reinhardt, and M. Ueda.
[1] D. Leibfried et al., Nature 438, 639 (2005).

[2] A. Ourjoumtsev et al., Nature 448, 784 (2007).

[3] H. Lee, P. Kok, and J. P. Dowling, J. Mod. Opt. 49, 2325 (2002).

[4] N. Gisin and H. Bechmann-Pasquinucci, Phys. Lett. A 246, 1 (1998).

[5] N. D. Mermin, Phys. Rev. Lett. 65, 1838 (1990).

[6] C. C. Gerry and R. A. Campos, Phys. Rev. A 68, 025602 (2003).

[7] A. N. Boto et al., Phys. Rev. Lett. 85, 2733 (2000); L. Pezzè and A. Smerzi, Eur. Phys. Lett. 78, 30004 (2007).

[8] G. Watanabe and C. J. Pethick, Phys. Rev. A 76, 021605(R) (2007).

[9] In Ref. [8], we pointed out that there is a one-to-one correspondence between the vortex-antivortex states discussed there and the Fock states with all particles in the right or left wells in the double-well potential.

[10] While this description is within the two-mode approximation, such a situation could be realized by setting the level spacing in each well much larger than the interaction energy per particle.

[11] G. J. Milburn, J. Corney, E. M. Wright, and D. F. Walls, Phys. Rev. A 55, 4318 (1997).

[12] A. Smerzi, S. Fantoni, S. Giovanazzi, and S. R. Shenoy,
Phys. Rev. Lett. 79, 4950 (1997).

[13] S. Fölling et al., Nature 448, 1029 (2007).

[14] D. Gordon and C. M. Savage, Phys. Rev. A 59, 4623 (1999).

[15] A. Micheli, D. Jaksch, J. I. Cirac, and P. Zoller, Phys. Rev. A 67, 013607 (2003).

[16] K. W. Mahmud, H. Perry, and W. P. Reinhardt, Phys. Rev. A 71, 023615 (2005).

[17] K. Mølmer and A. Sørensen, Phys. Rev. Lett. 82, 1835 (1999).

[18] F. Piazza, L. Pezzè, and A. Smerzi, Phys. Rev. A 78, 051601(R) (2008).

[19] M. H. Devoret, A. Wallraff, and J. M. Martinis, e-print arXiv:cond-mat/0411174.

[20] G. L. Salmond, C. A. Holmes, and G. J. Milburn, Phys. Rev. A 65, 033623 (2002).

[21] To obtain an almost perfect NOON state, one should slightly detune $\omega$ from Eq. (5).

[22] By modulating the amplitude $\Delta V$ of the tilt $\hat{V}_{\text {tilt }} \equiv$ $\Delta V \Delta \hat{N} / 2$ of the double-well potential, we do not obtain such a reduction of $T$ with a wide width in $\omega$ (G. Watanabe, to be published) unlike by modulating $J$ studied in the present work.

[23] J. H. Shirley, Phys. Rev. 138, B979 (1965); A. Eckardt, 
C. Weiss, and M. Holthaus, Phys. Rev. Lett. 95, 260404 (2005).

[24] We set $J(t)$ as, e.g., $J(t)=J\left[1+A\left(\sin \omega_{1} t+\sin \omega_{2} t\right)\right]$, with $\omega_{1}$ and $\omega_{2}$ corresponding to transitions between $|\Delta N|=$ $N$ and $N-2$, and between $N-2$ and $N-4$, respectively.

[25] D. R. Dounas-Frazer, A. M. Hermundstad, and L. D. Carr, Phys. Rev. Lett. 99, 200402 (2007); L. D. Carr, D. R. Dounas-Frazer, and M. A. Garcia-March, e-print arXiv:quant-ph/0610166

[26] The tilt in the double-well potential corresponds to the external rotation in the case of the vortex-antivortex system discussed in [8].

[27] Since the central value of $\omega$ of the wide resonance is slightly lower than that given by Eq. (5), we adjust $\omega_{0}$ in the fitting.
[28] To avoid excitations to higher energy levels by the modulation, $\hbar \omega$ should be much smaller than the level spacing in the well. For simplicity we consider $d_{\perp}=d_{z}\left(\equiv d_{w}=\right.$ $\left.\sqrt{\hbar / m \omega_{w}}\right)$, where $\omega_{w}$ is the frequency of each well. Since $\hbar \omega \sim U N$ from Eq. (5), and $U N / \hbar \omega_{w} \simeq \sqrt{2 / \pi}\left(a_{\mathrm{s}} / d_{w}\right) N$ [31], where $a_{\mathrm{s}}$ is the $s$-wave scattering length, that condition reads $N a_{\mathrm{s}} / d_{w} \ll 1$, which is not difficult to satisfy for $N=O(1)$.

[29] E. A. Burt et al., Phys. Rev. Lett. 79, 337 (1997).

[30] For these parameters, the condition for the tilt, $\Delta V N \ll$ $\Delta E_{N-2}$, reads $\Delta V / J \ll 0.6$, which can be easily satisfied.

[31] A. Smerzi, S. Fantoni, S. Giovanazzi, and S. R. Shenoy, Phys. Rev. Lett. 79, 4950 (1997). 